\title{
Complex Frontal Pneumosinus Dilatans Associated with Meningioma: A Report of Two Cases and Associated Literature Review
}

\author{
Sara Timms ${ }^{1}$ Raj Lakhani ${ }^{1}$ Steve Connor ${ }^{1}$ Claire Hopkins ${ }^{1}$ \\ ${ }^{1}$ Department of Rhinology, Guy's and St Thomas' Hospital, Great \\ Address for correspondence Sara Timms, MBBS, Department of \\ Maze Pond, London, United Kingdom \\ Rhinology, Guy's and St Thomas' Hospital, Great Maze Pond, London \\ SE19RT, United Kingdom (e-mail: sara.timms@doctors.org.uk). \\ J Neurol Surg Rep 2017;78:e97-e100.
}

\begin{abstract}
Introduction Pneumosinus dilatans (PSD) is a rare phenomenon involving the expansion of the paranasal sinuses, without bony destruction or a mass. Previously documented cases have demonstrated simple expansion of a solitary air cell. We present two unique cases of PSD in the presence of meningioma, in which complex new cells developed within the frontal sinus. One of the two patients developed associated sinus disease.

Case 1 A 28-year-old man presented with facial pain. A computed tomography scan showed an abnormally enlarged, septated right frontal sinus, not present on childhood scans. He underwent a modified endoscopic Lothrop approach to divide the septations, and his symptoms resolved.

Case 2 A 72-year-old woman presented with a 3-month history of headaches. Scans revealed a left frontal meningioma and multiple enlarged, dilated left frontal air cells. She had no clinical sinusitis and therefore was managed conservatively.

Keywords

- pneumosinus dilatans

- meningioma

- rhinosinusitis

- etiology

Conclusions PSD has been widely documented in association with fibrous dysplasia and meningioma. The most prevalent theory of the mechanism of PSD is of obstruction of the sinus ostium causing sinus expansion through a "ball-valve" effect. Our cases, which demonstrate septated PSD, suggest a more complex process involving local mediators and highlight the need to consider underlying meningioma in pneumosinus dilatans.
\end{abstract}

\section{Introduction}

Pneumosinus dilatans (PSD) is a rare phenomenon characterized by the expansion of one or more of the paranasal sinuses, without bony destruction or the presence of a mass in the sinus. ${ }^{1}$ It most commonly affects the frontal sinus, followed by the sphenoid, maxillary, and ethmoid sinuses. ${ }^{2}$

One proposed etiology of PSD is sinus obstruction with a "ball-valve" effect creating high pressure within the sinus, which leads to bone remodeling. There are reports of PSD associated with skull base meningioma adjacent to the sinuses. ${ }^{2-4}$ These reports postulate that the meningioma obstructs the sinus.

Meningiomas are tumors arising from the arachnoid mater, the middle layer of the meninges that surround the brain and spinal cord. They account for approximately $15 \%$ of all intracranial tumors. The World Health Organization classification denotes three subtypes: $80 \%$ of meningiomas are benign and are classified grade I, grade II meningiomas have atypical histology, and grade III meningiomas are aggressive anaplastic tumors that tend to metastasise. ${ }^{5}$ Meningiomas have been described throughout the cranial vault and are often asymptomatic. received

August 12, 2016

accepted

January 18, 2017
DOI http://dx.doi.org/

10.1055/s-0037-1600916.

ISSN 2193-6358.
(C) 2017 Georg Thieme Verlag KG Stuttgart · New York
License terms

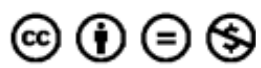



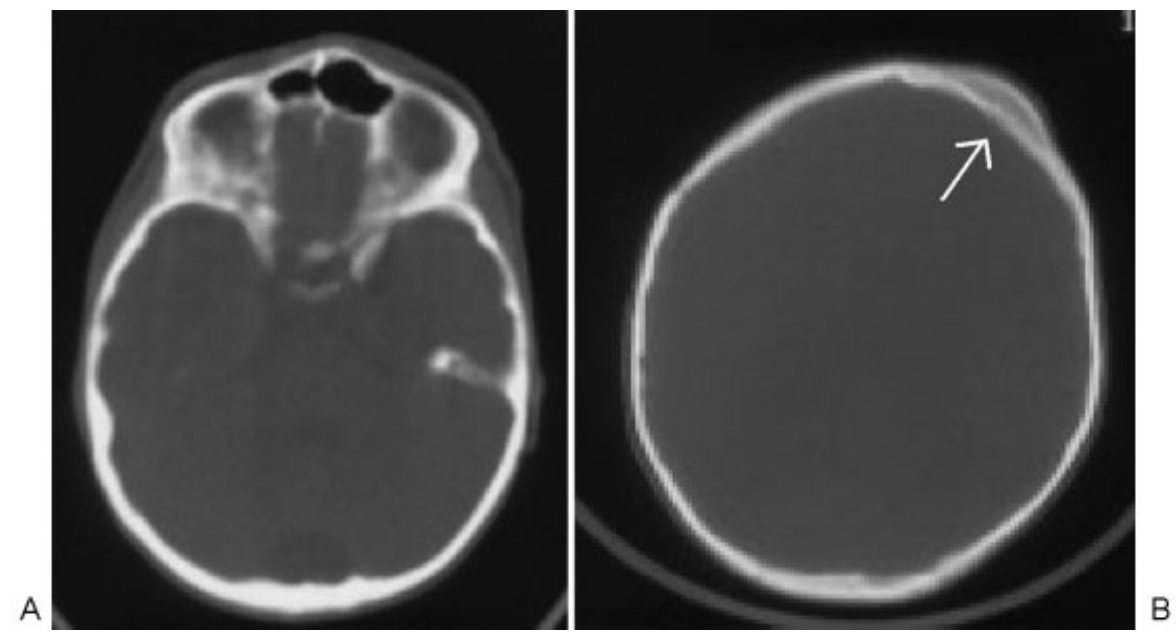

Fig. 1 Axial computed tomography of the head at the age of 7 years, (A) at the level of the frontal sinuses, demonstrating only minor asymmetric prominence of the left frontal sinus, and (B) more superiorly, demonstrating expansion of the left frontal bone (arrow) consistent with fibrous dysplasia.

Previously documented cases of PSD have demonstrated simple expansion of a solitary sinus air cell or the entire frontal or sphenoid sinus. We present two unique cases of PSD in the presence of meningioma, in which complex new cells developed within the frontal sinus. One of the two patients developed associated sinus disease.

\section{Case 1}

A 28-year-old man was referred with intermittent facial pain thought to be caused by recurrent acute frontal sinusitis. A computed tomography (CT) showed frontal and ethmoidal sinus disease. The history included multiple operations for fibrous dysplasia of the skull in childhood. More recently, he had been treated by the neurosurgeons for multiple meningiomas, including one in the left frontal region, resected 4 years previously, and a second meningioma in the olfactory groove, for which he had CyberKnife treatment 1 year prior to referral. The meningiomas were not in direct contact with the frontal sinus.

The images shown are CT head at 7 years of age ( $\mathbf{- F i g . 1} \mathbf{1}$ ), showing a thickened frontal bone secondary to fibrous dysplasia, and anatomically normal frontal sinuses with a single midline septation; CT sinuses at 23 years of age, showing frontal PSD with multiple frontal sinus cells (-Fig. 2); and a further CT 3 years later in which the frontal sinus is opacified during an episode of acute sinusitis (-Fig. 3).

Recurrent infective episodes were confirmed with endoscopic examination demonstrating purulence in the middle meatus, with fever and raised inflammatory markers during acute episodes. Conservative management failed to relieve his symptoms. The patient underwent a modified endoscopic Lothrop approach to allow excision of all the bony septations within the frontal sinus, as it was felt that a simple frontal sinus approach would not relieve the anatomical obstruction
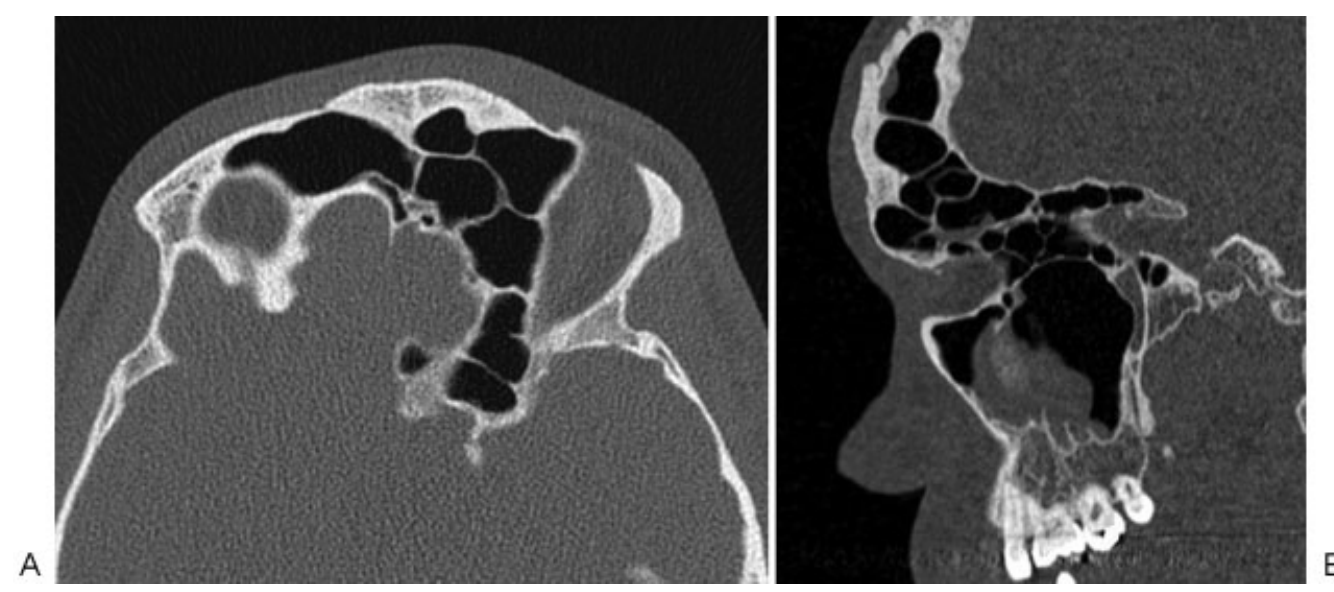

Fig. 2 Axial (A) and sagittal (B) computed tomography images of the frontal sinuses at the age of 23 years, demonstrating marked increase in the pneumatization of the left frontal sinuses and supraorbital air cells. Multiple internal septations are evident (with expansion of the orbital roof and extension posteriorly to the anterior clinoid). 


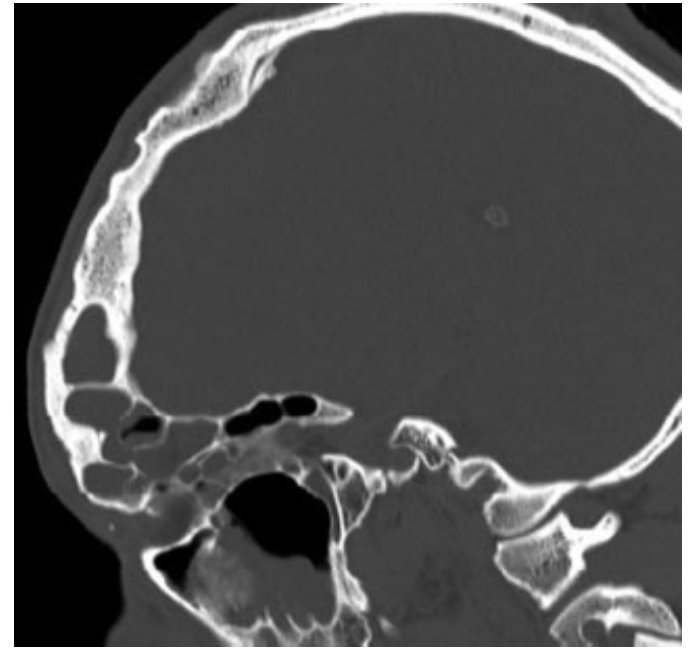

Fig. 3 Sagittal computed tomography image of the frontal sinuses at a similar level to - Fig. 2B. There is interval opacification of the left frontal sinus and additional frontal and supraorbital air cells. Note the diffuse expansion of the left frontal bone (with expansion of the diploic space at the site of the fibrous dysplasia and irregularity of the outer table of the frontal bone at the site of previous biopsy).

associated with the complex air cells. Images taken during the procedure ( $\boldsymbol{- \text { Fig. }}$ 4) show some of the multiple left frontal sinus air cells viewed with a 30-degree rigid nasendoscope. At 1 year postoperative follow-up, he had no further facial pain or nasal symptoms and was discharged.

\section{Case 2}

A 72-year-old woman presented to the neurology department with a 3-month history of headaches. A magnetic resonance imaging (MRI) performed as part of the investigations showed a right frontal meningioma in contact with a grossly septated and dilated right frontal sinus. There was a second meningioma noted in the left cavernous sinus region. These images were reviewed at the skull base multidisciplinary meeting, where it was decided that the lack of clinical symptoms or radiological evidence of sinus disease negated the need for sinus surgery at present. A conservative approach for the meningioma is currently being taken, with no further growth and with resolution of the headaches.

This patient has been fit and well for many years and had had no previous imaging of her head until her headaches arose. - Figs. $\mathbf{5}$ and $\mathbf{6}$ are her CT and MRI scans, respectively, showing the frontal meningioma and new frontal sinus air cells.

\section{Discussion}

The frontal sinus is a paired paranasal sinus in the frontal bone, located superomedially to the orbit. PSD was first documented by Benjamins in 1918, who described it as an abnormally dilated frontal sinus containing air alone. ${ }^{6}$ Lloyd documents a case series of six patients with PSD, in which
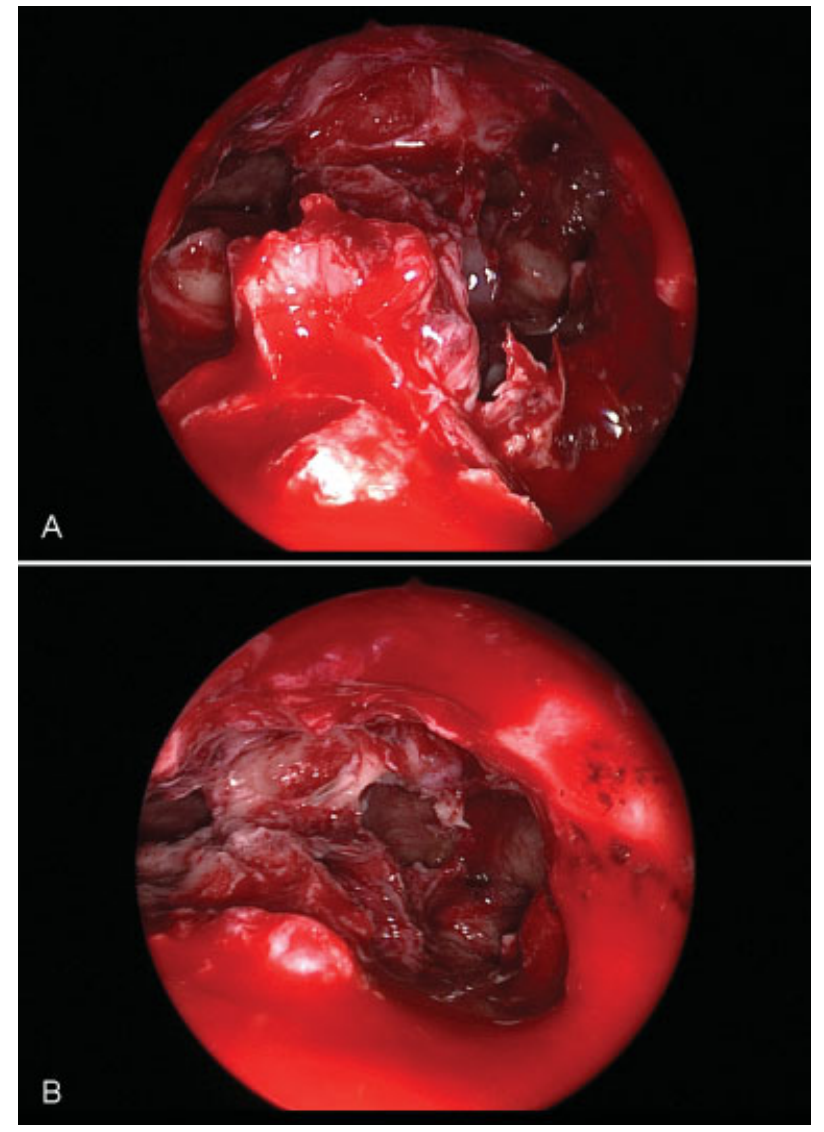

Fig. 4 (A,B) Intraoperative views of the frontal sinus using a 30 degree rigid nasendoscope. Multiple air cells are visible.

three have concurrent meningioma and three are patients with fibro-osseous disease. ${ }^{7}$ We suspect that his previous fibrous dysplasia may be the more likely explanation for our male patient's PSD (case 1) since his meningioma is not in direct contact with the frontal sinus. However, it may also imply a link between meningioma and PSD that is not purely mechanical.

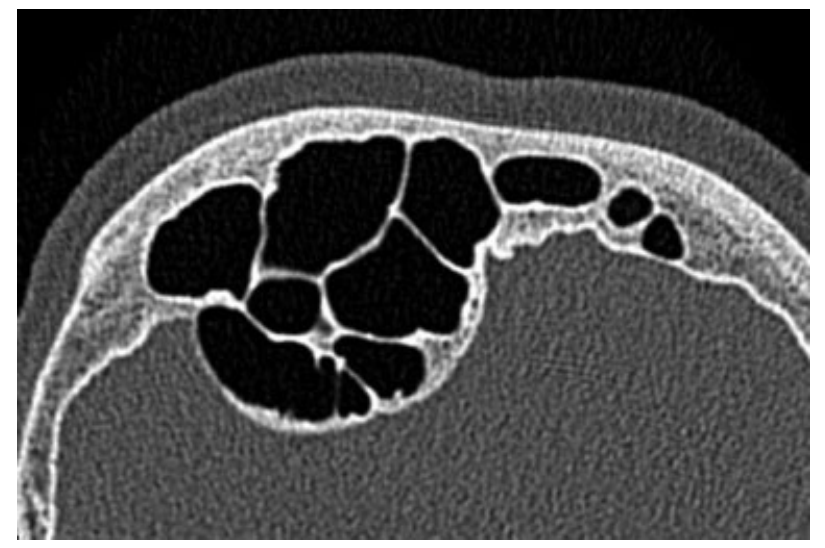

Fig. 5 Axial computed tomography showing marked focal expansion of the right frontal sinus with numerous septations and additional air cells. There is minor thinning of the dorsal wall of the frontal sinus. 

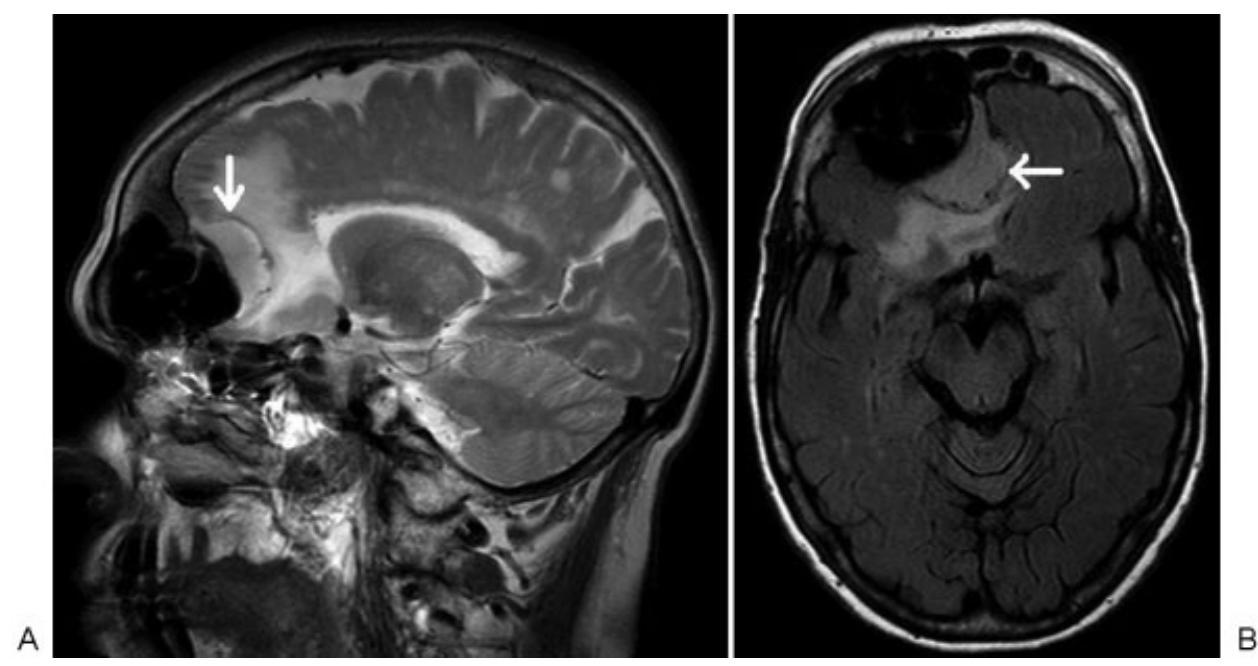

Fig. 6 Sagittal T2 image (A) and axial fluid-attenuated inversion recovery image (B) revealing the expanded right frontal sinus to be immediately adjacent to the right frontal meningioma (arrows). Adjacent hyperintense edema and mass effect are demonstrated in the frontal white matter.

Our cases are unique in the current literature as previous cases have reported expansion of a solitary cell and none have described de novo sinus cell formation. While we cannot be sure that complex cell formation was not present prior to meningioma formation in case 2, the availability of childhood scans in case 1 demonstrates that the cells certainly formed later in his case, after normal embryological development of the sinus, and we postulate that the same is true of our other case. Although the frontal sinuses are not fully formed until late adolescence, ${ }^{8}$ in our first patient, they are well developed in the scan at 7 years of age, and no abnormal frontoethmoidal sinus cells can be seen.

The etiology of PSD is unknown, particularly in association with meningioma, although various theories have been proposed. Most commonly, it is suggested that the meningioma may obstruct the sinus ostium, resulting in a "ballvalve" system in which the volume of air within the sinus gradually increases. This theory is consistent with a single dilated air cell seen in previously reported cases.

Alternatively, the meningioma may have a less direct influence on the sinus wall. This may be hormonal; vascular, as a result of increased dural blood flow; or related to local cytokines that alter the balance between osteoblastic and osteoclastic activity. ${ }^{3,4,9}$ The two cases we present add to the evidence that meningioma and fibro-osseous disease may cause PSD. Additionally, the formation of new air cells within the sinus in our two cases would suggest that a simple ballvalve effect causing expansion of the sinus is less likely, as this would lead to dilation of a single anatomical sinus cell. We believe that these two cases are able to give further insight into the etiology of PSD, implicating local mediators that stimulate bone turnover and remodeling.
These cases also highlight the importance of considering and excluding an underlying meningioma in patients with PSD, which may present simply with cosmetic changes in facial appearance. ${ }^{3}$

\section{Funding}

The authors did not receive any financial support for the work in this article.

\section{References}

1 Miller NR, Golnik KC, Zeidman SM, North RB. Pneumosinus dilatans: a sign of intracranial meningioma. Surg Neurol 1996; 46(05):471-474

2 Gibbons BA, Miele WR, Florman JE, Heilman CB, Horgan MA. Pneumosinus dilitans and meningioma: a case series and review of the literature. Neurosurg Focus 2011;30(05):E13

3 Adams WM, Jones RI, Chavda SI, Pahor AL, Taifa KT. Pneumosinus dilatans: a discussion of four cases and the possible aetiology. Rhinology 1998;36(01):40-42

4 Parizel PM, Carpentier K, Van Marck V, et al. Pneumosinus dilatans in anterior skull base meningiomas. Neuroradiology 2013; 55(03):307-311

5 Lumenta CB, Di Rocco C, Haase J, Mooij JJA. Neurosurgery. Heidelberg. Germany: Springer; 2010:96

6 Benjamins CE. Pneumosinus frontalis dilatans. Acta Otolaryngol 1918;1:412-444

7 Lloyd GA. Orbital pneumosinus dilatans. Clin Radiol 1985;36(04): $381-386$

8 Duque CS, Casiano RR. Surgical anatomy and embryology of the frontal sinus. In: Kountakis SE, Senior BA, DrafW, eds. The Frontal Sinus. Heidelberg. Germany: Springer; 2005:21-31

9 Urken ML, Som PM, Lawson W, Edelstein D, Weber AL, Biller HF. Abnormally large frontal sinus. II. Nomenclature, pathology, and symptoms. Laryngoscope 1987;97(05):606-611 